\section{RESILIENCIA COMO FENÓMENO SOCIAL Y PROCESO EN UN CONTEXTO DE DESASTRE: HORIZONTE TEÓRICO DESDE KANT Y SIMMEL}

Jorge Luis Montero Rodríguez ${ }^{1^{*}}$

\section{RESUMEN}

El proceso de desastre desvela varias situaciones críticas: la penuria post-impacto, la espera en el albergue y la reubicación poblacional. Dentro, la resiliencia social se vuelve efectiva en las acciones de afrontamiento, recuperación, adaptación y transformación de la sociedad. Ella evidencia la acción de los agentes sociales e instituciones para contrarrestar los estados negativos que viven; de esta manera se constata que una sociedad es resiliente. Pero, definida únicamente desde la capacidad o propiedad de los sistemas, no da seguridad de la eficiencia en las acciones ejecutadas durante la crisis. Por ende, el presente artículo inicia con las diversas definiciones sobre el concepto $y$, en un segundo apartado, utiliza varios reportes de investigaciones donde el comportamiento resiliente de los agentes evidencia a la resiliencia como fenómeno social que, en remisión a Kant, es percibido y comprendido en su manifestación activa, y que, basado en la teoría de Georg Simmel, posee analogía analítica al conflicto. Esta revisión bibliográfica permitió calzar el método heurístico de reconocimiento de la resiliencia como proceso y fenómeno social. A consecuencia, se propuso el uso del verbo resilir como concepto categorial que refiere a todas las acciones resilientes.

\section{PALABRAS CLAVE}

Resiliencia, Resiliencia social, Fenómeno social, Desastre, Conflicto

RESILIENCE AS A SOCIAL PHENOMENON AND PROCESS IN A DISASTER CONTEXT: THEORETICAL HORIZON FROM KANT AND SIMMEL

\section{ABSTRACT}

Disaster process shows several critical situations: post-impact damage, pressure on shelter provision, and sometimes resettlement. Social resilience becomes relevant in coping, recovery, adaptation and transformation actions by society. Social resilience evidences both action of social agents and institutions to overcome the negative state they face. Through this process, society can be identified as resilient. But, if social resilience is only defined as ability or property of the social systems, then there is no certainty about efficient actions to overcome a crisis. Therefore, this paper aims to demonstrate resilience as a social phenomenon that, according to Kant, it is perceived and understood from its active manifestation, and, based on Simmel's theory, it has an analytical analogy to conflict. The analysis starts about different definitions of social resilience and, in the second section, uses several investigations about resilient behavior of individuals and groups to demonstrate the above mentioned. The literature review enabled to fit the heuristic method of recognition of resilience in as a process and social phenomenon. Subsequently, the paper proposes the use of resile as a categorical concept referred to all resilient actions.

\section{KEYWORDS}

Resilience, Social resilience, Social phenomenon, Disaster, Conflict
1. Universidad de Guadalajara, Guadalajara, México.

*Autor de correspondencia: jorgelmontero86@gmail.com

\section{RECIBIDO}

1o de diciembre de 2019

\section{ACEPTADO}

20 de diciembre de 2019

\section{PUBLICADO}

1 de enero de 2020

\section{Formato cita}

Recomendada (APA): Montero Rodríguez, J.L. (2020). Resiliencia como fenómeno social y proceso en un contexto de desastre: Horizonte teórico desde Kant y Simmel. Revista de Estudios Latinoamericanos sobre Reducción del Riesgo de Desastres REDER, 4(1), pp.105-118.

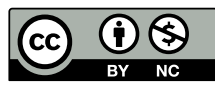

Todos los artículos publicados en REDER siguen una política de Acceso

Abierto y se respaldan en una Licencia CreativeCommons Atribución-NoComercial 4.0 Internacional.

Revista de Estudios Latinoamericanos sobre Reducción del Riesgo de Desastres (REDER)

Diseño: Lupe Bezzina Tipografía: Hospital 


\section{INTRODUCCIÓN}

El desastre asociado a fenómenos naturales no refiere únicamente a un evento momentáneo de experiencias extraordinarias cual hecho puntual en la vida de las sociedades humanas. El desastre posee multiespacialidad, así como, duración corta o prolongada que dependen de la efectividad de las acciones preventivas y de la eficacia de las recuperativas posteriores. Las primeras están encaminadas a mitigar y eliminar las vulnerabilidades construidas socialmente para minimizar los riesgos. Las acciones recuperativas abordan los efectos dañinos del desastre para tratar de contrarrestarlos y restaurar la estabilidad social. Cuando la gestión del riesgo y los planes preventivos son efectivos, la magnitud de los daños es mínima y el período de crisis post-impacto puede ser corto. Adicionalmente, si los programas de recuperación son eficaces, ese tiempo corto es reducido aún más y el final de la crisis es inmediato. En uno u otro período, se pueden dar varias situaciones críticas, cual una de ellas es el proceso de reubicación poblacional post-impacto. Estos procesos de reubicación no son centrales en este trabajo, pero se utilizarán recurrentemente como ejemplo para enfatizar ciertos puntos.

Usualmente, las reubicaciones agravan la penuria que sufren los damnificados porque suponen el abandono de la forma de vida anterior $y$, como han reconocido varios investigadores (Oliver-Smith, 1991; 1994; Macías, 2009; Briones, 2010; Arévalo, 2012), son proclives al fracaso cuando no toman en cuenta los aspectos socioculturales de habitar y vivir de las comunidades.

La suposición consiguiente es que los proyectos de reubicación tienen a la adaptación como estrategia (Warner et al., 2015; McAdam, 2015), no así a la resiliencia social. Ellos son asumidos gubernamental y administrativamente como una solución al problema del desastre y la crisis subsiguiente, no como disrupciones o situaciones críticas en sí mismos, lo cual distingue entre adaptación y resiliencia. Tal diferencia es clara en las operaciones de cambios estructurales, funcionales y de estados en los sistemas complejos, donde las escalas esgrimen dicha distinción (Gunderson \& Holling, 2002). En los sistemas sociales que viven la complejidad del proceso de desastre, la resiliencia juega un papel importantísimo.

Definida de diversas maneras según el campo disciplinario que la trabaje -sea desde la física de materiales, la ingeniería, la biología y ecología, o desde las ciencias sociales como en la psicología y la sociología- su núcleo fuerte de definición está en la capacidad o conjunto de capacidades (Faulkner et al., 2018) que un sistema posee y produce en procesos conservativos, adaptativos y/o transformativos que le confieren una propiedad única de existencia y supervivencia (Holling, 1973; Walker et al., 1997; Gunderson, 2000; Carpenter, 2014; Uriarte, 2013; Keck \& Sakdapolrak, 2013). La resiliencia social, por tanto, no queda fuera de este núcleo, más bien se enriquece con factores económicos, políticos, culturales y tecnológicos.

En el 2008, Cutter et al. la definieron como:

"la capacidad del sistema social para responder y recuperarse de los desastres que incluye las condiciones inherentes al sistema que le permite absorber impactos, afrontar el evento y sus efectos posteriores, así como también, llevar procesos adaptativos que facilitan su capacidad para reorganizarse, cambiar y aprender en respuesta a una amenaza."

(Cutter et al., 2008, p. 599)

Asimismo, Ainuddin y Routry (2013) ofrecieron un compendio de diversas definiciones, incluyendo la ofrecida por Cutter et al. enunciada arriba. De los autores citados, la gran mayoría aluden a la resiliencia social en términos de capacidad y solo uno -el grupo de investigadores de MCEER (2007)- incluye la dimensión activa de las acciones sociales.

En su resumen, la resiliencia social aparece asociada a ciertos estados, condiciones y resultados como son: la resistencia, organización social, preservación, restauración, administración, adaptación, recuperación y fortalecimiento de funciones y estructuras sociales. Conceptos como: estrés, perturbación, adversidad y desastre tienen sentidos causales; y, paralelamente, comparecen factores como: la utilización de recursos físicos y económicos, la vitalidad socieconómica, la organización y la funcionalidad de la sociedad afectada. Además, es entendida como un proceso vinculante de capacidades adaptativas en trayectoria de cambio positivo, así como una red sostenible de sistemas físicos y sociales capaz de manejar las diversas crisis actuales y futuras (Tobin, 1999; Adger, 2000; Paton \& Fohnston, 2001; Godschalk, 2002; Bruneau et al., 2003; Twigg, 
2007; Maguire \& Hagan, 2007; MCEER, 2007; Cutter et al., 2008; Norris et al., 2008; Kulig et al., 2008; UNISDR, 2009, citados por Ainuddin \& Routry, 2013). Todo esto evidencia un espectro circunscripto a la noción de capacidad, originaria de la física de materiales y la psicología (Forés \& Grané, 2008).

Dentro de este enfoque sostenible de red, Contreras y Beltrán $(2015$, p. 86) extrajeron a la resiliencia, "como la condición que posee un territorio para sobreponerse ante efectos adversos e inesperados". La interconexión entre los sistemas físicos y sociales toma aquí la figura conceptual: territorio. Y es también el territorio esa categoría operativa sistémica conveniente en la gestión de riesgo donde la resiliencia social y la urbana quedan dentro del disaster resilience (MCEER, 2007). Los elementos fundamentales del disaster resilience son: el contexto, la perturbación, la capacidad de lidiar con la perturbación, y la reacción ante la perturbación (DFID, 2011).

Otro pesquisaje exhaustivo fue realizado por Keck y Sakdapolrak (2013, p. 8) quienes concluyeron que: "todas las definiciones de resiliencia social se refieren a entidades sociales -sean individuos, organizaciones o comunidades- y a sus capacidades (abilities o capacities) para tolerar, absorber, afrontar y ajustarse a las amenazas ambientales y sociales de diversos tipos". Esta noción no está muy distante de los elementos significantes enunciados antes. El descubrimiento excepcional de estos autores fue que dicho concepto ha sido una matriz guía compuesta por: capacidades de afrontamiento, capacidades adaptativas, y capacidades transformativas; de modo que, en general, podría ser enmarcado entre la persistencia, la adaptación y la transformación de los sistemas sociales.

Por ende, a partir de estos enmarques, cuando las "actividades recuperativas son realizadas en vías de minimizar la disrupción social y también mitigar los efectos de futuros desastres" (MCEER, 2007, p. 4), podemos discernir entre acciones de resistencia, de adaptación y/o de transformación, como igual Uriarte (2013) remitió a ello cuando reconoció tres categorías fundamentales: la resiliencia social como estabilidad, como recuperación y como transformación.

No obstante, a pesar de esa remisión implícita a diversas acciones con potenciales de cambio, la mayor alusión del concepto ha estado circunscripta a la capacidad (Uriarte, 2013; Prieto, 2013). A pesar de poseer actividad, para Uriarte $(2013$, p. 10) la resiliencia social no deja de ser "la capacidad del sistema social y de las instituciones para hacer frente a las adversidades y para reorganizarse posteriormente de modo que mejoren sus funciones, su estructura y su identidad". Mientras, Prieto (2013, p. 25) distinguió que, "en términos generales, es la capacidad de una entidad social a responder positivamente a la adversidad".

El peso de esta circunscripción está presente en los marcos legales y operativos de organizaciones mundiales y gubernamentales locales siempre con perspectivas preventivas. Tal es el caso de la definición ofrecida por la United Nations Office for Disaster Risk Reduction: "La capacidad de un sistema, comunidad o sociedad [...]" (UNDRR, 2020); o, la enunciada en la Ley General de Asentamientos Humanos (2016, p. 4): "la capacidad de un sistema, comunidad o sociedad [...]"; definida igual en la Ley General de Protección Civil mexicana (2017). En la primera se aclara que los tres niveles de gobierno e instancias afines están orientados a fortalecer e incrementar la resiliencia con propósitos preventivos, a la vez que coordinan la creación de ciudades y asentamientos humanos resilientes. Mientras que, en la segunda, se indica que las políticas públicas a generar sobre protección civil deben tener como prioridad "el fomento de la participación social para crear comunidades resilientes, y por ello capaces de resistir los efectos negativos de los desastres, mediante una acción solidaria [...]" (p. 7). De modo que la prioridad para las entidades públicas es fomentar y fortalecer las capacidades de resiliencia social, desestimando así el papel gubernamental en el momento posterior de reacción al desastre.

Tal fue el caso del proyecto de reubicación por desastre Ciudad Rural Sustentable Nuevo Juan de Grijalva en Chiapas, México, donde las leyes mencionadas tuvieron incidencia en el marco legal de la implementación de dicha política pública. Por demás, aunque el concepto de resiliencia social no fue utilizado en el programa operativo, las estrategias de capacitación a la población reubicada poseyeron el principio resiliente de favorecer la adaptación al cambio de hábitat para superar los efectos negativos del desastre. Sin embargo, como bien identificó Arévalo (2012), la mala atención a los elementos socio-culturales condujo al fracaso.

Arévalo (2012) estudió los efectos de las prácticas sociales de los reubicados luego del otorgamiento del nuevo asentamiento que ofrecía nuevas oportunidades de desarrollo y bienestar. Así, fue propuesto un novedoso esquema productivo-económico que exigió de los habitantes un afrontamiento diferente al del evento catastrófico vivido antes. Se recurrió entonces al potencial 
adaptativo de la población previamente instruida y capacitada para la nueva realidad laboral y residencial, pero el resultado fue opuesto al esperado. Los habitantes no lograron ascender tales capacidades resilientes al nivel práctico, por lo que fueron incapaces de insertarse en la nueva dinámica económica impuesta, diferente a la dinámica agrícola conocida. Ergo, a pesar de haberse dado un cierto grado de adaptación al medio, el fracaso del proyecto fue palpable cuando los reubicados optaron por retornar a sus antiguos hábitats y negar el nuevo.

Con este ejemplo se demuestra la operatividad institucional de la resiliencia como fomento y fortalecimiento de capacidades determinadas, no como activación y cooperación en acciones sociales. De modo que, no necesariamente las capacidades alcanzadas por una comunidad o sociedad para encarar las dificultades dentro del proceso de desastre pasan a ser efectivas en la resistencia, adaptación y/o transformación sin acciones que lo hagan posible. Si un grupo social, comunidad o sociedad no logra superar las situaciones críticas del proceso de desastre, entonces no son sistemas resilientes por más capacitados que estén.

Por esta razón, es necesario incluir explícitamente la vertiente activa en la definición de resiliencia social que tribute a su adecuada teorización y operación, pues, como bien problematizó Abend (2008), la multiplicidad de nociones y confusiones semánticas sobre un mismo concepto deparan en disímiles formas de abordaje y de operacionalizarlo. Frecuentemente se cae en errores al trabajar con un término que refiere imprecisamente a algo de interés sociológico y político; con ello aparecen problemas práctico-políticos a partir de los desacuerdos y disensos que la imprecisión provoca.

El objetivo del presente artículo es evidenciar el carácter activo de la resiliencia social con base en estudios ecológicos y sociológicos. De esta manera, sin negar la circunscripción de la resiliencia en la capacidad, pertinente en la gestión de riesgo, la intención es poner en evidencia su realidad fenoménica en el proceso de desastre. Para tal efecto, se presentan, en un primer apartado, ejemplos de investigaciones empíricas movilizadas por esta circunscripción. Conjuntamente, se citan aquellas centradas en el comportamiento resiliente de sistemas ecológicos y sociales donde los agentes muestran actividad para contrarrestar las diversas situaciones críticas. La intención es moldear un escenario empírico en donde pueda ser aplicado el método heurístico del reconocimiento (Gigerenzer, 2008) desde una perspectiva filosófica (Ricoeur, 2006). Las teorías de Kant y Simmel sirven para tejer una hebra teórica de la resiliencia social como fenómeno social dentro del conflicto. El proceso de desastre es contextualizado así en la noción sociológica del conflicto.

Por último, el trabajo incita a una discusión sobre las proposiciones teóricas y semánticas derivadas del análisis; todo ello con la pretensión de abonar a la teorización identitaria de la resiliencia social para su posterior operacionalización y teorizaciones particulares.

\section{HACIA EL SABER DE QUÉ ES LA RESILIENCIA SOCIAL}

Todos los estudios concernientes a la resiliencia social evidencian una cosa ineluctable: ella pertenece a la realidad de las sociedades humanas $y$, por ende, adquiere sentido social para el ser humano. El que tenga sentido y realidad, la hace susceptible a definición, por ello es propensa a reconocimiento y explicación científicos. La reconocemos cuando identificamos su naturaleza y función; y la explicamos cuando la hacemos operable, gestionable y planificable. Los efectos o resultado que ella deja en la realidad social permiten reconocerla y explicarla, por ello hay que mirar hacia las implicaciones sociales, culturales, políticas, económicas y tecnológicas que tiene, y así, dilucidar cómo debemos reconocerla y explicarla para que dichas implicaciones sean positivas.

Del ejemplo de Arévalo (2012) es posible extraer una parte de las implicaciones políticas y administrativas que en la esfera operativa tiene el enfoque de la resiliencia social focalizado solo en las capacidades, recursos o propiedades. El caso de Nuevo Juan de Grijalva evidenció un programa político fallido con deficiencias y repercusiones administrativas en la programación residencial y económica del nuevo asentamiento. No bastó con ofrecer viviendas básicas, ni proporcionar un esquema productivo-económico novedoso para que los reubicados actuaran en favor de sus propias adaptaciones. El recelo a realizar las nuevas prácticas económicas catalizó el rechazo y abandono del nuevo asentamiento.

Esto demuestra que la gestión previa de capacidades y recursos resilientes no es nada sin su aplicabilidad posterior en las acciones resilientes efectivas en la etapa de recuperación 
post-impacto. De no ser así, no es posible reconocer a las comunidades reubicadas como resilientes, ni tampoco se llega a ese reconocimiento si no hay involucramiento activo de los damnificados e instituciones en la rehabilitación o reconstrucción post-impacto -véase estudio realizado por Contreras y Beltrán (2015).

Dentro del espectro circunscripto a la capacidad, varias investigaciones han tratado de proveer indicadores, marcos analíticos, modelos, estrategias y métodos de medición. Al respecto Marshall y Marshall (2007) identificaron cuatro características de la resiliencia en pescadores de Queensland sometidos a hipotéticos cambios en las políticas de pesca, a saber: 1) la percepción del riesgo asociado al cambio, 2) la capacidad de planificar, aprender y reorganizarse, 3) la capacidad de hacer frente a la dificultad, y 4) el nivel de interés en el cambio. Como resultado, los autores abogaron por incluir la percepción del riesgo en los modelos conceptuales de resiliencia social, y proporcionaron un marco operativo estandarizado para definirla y cuantificarla a priori al afrontamiento o a la adaptación.

Dos años después, Ross, Cuthill, Maclean, Jansen y Witt (2010) estudiaron a seis poblaciones de la misma región australiana y enunciaron seis indicadores o atributos fundamentales: 1) conocimiento, habilidades y aprendizaje, 2) redes comunitarias, 3) conexión de las personas con el lugar, 4) comprometimiento del gobierno, 5) economía diversa e innovadora, e 6) infraestructura comunitaria. Con esto formularon un marco de monitoreo que permitiría orientar las agencias de planificación y evaluación de la resiliencia social ante posibles desastres.

Por su parte, Faulkner et al. (2018) pusieron bajo el lente introspectivo a las poblaciones costeras de Boscastle y Wadebridge en Cornwall, Reino Unido. Su premisa fue que la resiliencia es una propiedad socialmente contingente de los sistemas socio-ecológicos. Por ello, el estudio estuvo enfocado en el conjunto de capacidades detectables, evaluables y medibles en una población preparada para afrontar un desastre. Tales capacidades fueron enunciadas como sigue: 1) arraigo al lugar, 2) liderazgo, 3) cohesión y eficacia comunitaria, 4) redes comunitarias, 5) conocimiento y aprendizaje. Estas son capacidades interconectadas que varían según la comunidad.

Sintéticamente, los tres estudios abordaron una resiliencia previa al desastre, posiblemente efectiva en el período de recuperación, pero sin decir nada al respecto. Para ello, los investigadores utilizaron encuestas y entrevistas que registraron criterios y opiniones individuales acerca de ciertas capacidades y recursos colectivos para afrontar, superar y aprender de las adversidades, sin expresar la activación certera y efectiva de acciones que lo hicieran posible. La suposición es que la planificación, coordinación y dirección de tales acciones quedan implícitas en la gestión de capacidades y recursos en una resiliencia social previa. Esta referencia a la acción implícita es rastreable en los estudios ecológicos, socio-ecológicos y sociológicos más avanzados en su análisis que los antes mencionados.

Por ejemplo, Holling (1973) quien primero utilizó el término resiliencia en sistemas de organismos vivos (Gunderson, 2000), expuso que, en el bosque canadiense de coníferas, las interacciones entre los árboles, las orugas del abeto y los depredadores de estas determinaron variados momentos de inestabilidad y estabilidad en el ecosistema. Por un lado, la eclosión esporádica de orugas que se alimentan del abeto maduro provocó que la permanencia de dichos árboles quedara afectada, propiciando que píceas y abedules imperaran. Dichas eclosiones sucedieron en secuencias de años secos. Mientras no ocurrieron, los depredadores y parásitos impactaron en el decrecimiento de la población de orugas (Morris, 1963; Baskerville 1971; Wellington 1952, citados por Holling, 1973).

La coincidencia de las temporadas secas con la abundante flora de abetos maduros favoreció el aumento de las orugas que escaparon del control de sus enemigos y, con este crecimiento, bajó el número de abetos y aumentó el de píceas y abedules. Cuando no hubo brote de orugas, los abetos prosperaron reprimiendo a las demás coníferas.

En otras palabras, si las orugas no se hubieran reproducido y no hubieran resistido la depredación a la que estaban sometidas y, además, no se hubieran alimentado de los abetos, entonces las píceas y los abedules no hubieran podido crecer y prevalecer en el bosque. Por el contrario, si los enemigos de las orugas no se hubieran alimentado de ellas y los abetos no se hubieran regenerado y crecido con el tiempo, entonces las otras coníferas hubieran dominado perennemente el ecosistema. Sin tales indicios de acciones en los organismos vivos, Holling (1973) no hubiera concluido que los comportamientos de estabilidad y resiliencia observados conforman 
un ciclo límite estable de gran amplitud en el bosque de coníferas cual sistema altamente inestable con enorme capacidad de recuperación.

Por su parte, Walker, Langridge y Mcfarlane (1997) estudiaron el proceso de recuperación de la pradera de sabana al norte de Australia mediante un experimento que duró ocho años donde alteraron la abundancia del pasto perenne. Al final descubrieron que la sabana manifestó diversos patrones de recuperación según las condiciones posteriores a las alteraciones. Por lo que, observando el comportamiento del pasto y de los organismos vivos cohabitantes, calificaron de resiliente al césped de la sabana.

Gunderson (2000) se hizo eco de este estudio y de dos casos más: los ecosistemas de los lagos poco profundos norteamericanos y los humedales de Everglades, para fundamentar la multiplicidad de estados estables que se dan en fenómenos ecosistémicos donde se manifiesta la resiliencia de manera medible según la magnitud de la perturbación que el sistema puede absorber en cada uno de los pasos de dichos estados.

Consideró a la resiliencia como una propiedad emergente relacionada con el comportamiento auto-organizado de los ecosistemas. De los lagos remarcó la interacción entre algas, fitopancton, aves y peces; de los humedales resaltó la nutrición de las plantas como factor determinante de los variados estados de estabilidad del ecosistema.

En suma, Holling (1973), Walker et al. (1997) y Gunderson (2000) enunciaron una resiliencia propia de sistemas complejos a partir de estudiar los comportamientos de sus agentes, y por ende las acciones e interacciones dadas. Por demás, en la mayoría de estos casos hubo incidencia de la acción humana, ya fuera para generar las perturbaciones o para favorecer en la recuperación del sistema socio-ecológico. De aquí es posible decir que la actuación humana es determinante en las manifestaciones resilientes de los sistemas socio-ecológicos, tal y como los efectos de los agentes naturales lo son en la resiliencia social de los sistemas sociales.

Al respecto, Carpenter (2014) realizó un análisis del comportamiento resiliente, en el proceso de recuperación post-impacto, de poblaciones estadounidenses afectadas en el 2005 por el huracán Katrina. La autora presupuso que las redes sociales, favorecidas por determinadas condiciones residencial-urbanas, aumentaban la resiliencia comunitaria. Su finalidad fue prescribir un adecuado actuar socio-gubernamental para la construcción del entorno urbano. A propósito, diseñó una metodología de investigación que le permitió medir la capacidad de adaptación de la población luego del desastre a través de las restauraciones hechas al entorno residencial como evidencia de recuperación a largo plazo.

El estudio demostró que, después de cinco años del huracán, ciertas características de la recuperación del entorno residencial poseían fuerte asociación con la interacción social y las redes sociales. Por ejemplo, la restauración de la densidad de intersecciones urbanas y de las vías mejor conectadas tuvo marcada vinculación con la conectividad, la accesibilidad y el tránsito peatonal socializante. También, la recuperación de locales históricos, templos, escuelas, restaurantes, centros recreativos y comunitarios incidió positivamente en las redes sociales.

En conclusión, Carpenter (2014) aseguró que las comunidades estudiadas manifestaron un comportamiento resiliente visible en el retorno a los hogares y físicamente evidente en la reconstrucción y rehabilitación del entorno residencial. Las características de este último favorecieron las redes sociales base y apoyaron al capital social. Con esto validó desde la acción su asunción de una resiliencia comunitaria cual capacidad de las poblaciones afectadas de recuperarse luego del evento catastrófico para mantener las mismas relaciones sociales que le permitieron persistir; y, con tal criterio, recomendó prestar atención a la gestión y planificación urbana como vía para mejorar la resiliencia general y reducir las vulnerabilidades.

Al sur de América, Herrera y Rodríguez (2016) abordaron la resiliencia comunitaria como la capacidad del sistema social para responder ante perturbaciones provocadas por agentes naturales que alteran panárquicamente la dinámica del sistema, cuyos factores determinantes son las capacidades de aprendizaje, de autoorganización, adaptación y transformación del sistema. Su marco analítico fue la resiliencia social activa dentro del ciclo adaptativo de los sistemas complejos en el caso de la ciudad Baños de Agua Santa en Ecuador donde, en el 1999, ocurrió la erupción del volcán Tungurahua.

Ante la crisis social y económica que provocó el desastre, la población volcó su capital cognitivo, económico y social hacia el reajuste de las actividades productivas. Los pobladores renovaron 
el sistema económico basado en el turismo religioso y de descanso, a un turismo de aventura, excursiones y deportes extremos favorecidos por las condiciones ecológicas y orográficas de la zona. Esto fue favorecido por la especialización del sector, la capacidad emprendedora de habitantes y empresarios, la estructura empresarial diversa y equilibrada, el fuerte tejido social, y la capacidad de aprendizaje de la sociedad. Así, la ciudad Baños evidenció una eficaz adaptación del sistema socioeconómico y una efectiva auto-organización recuperativa.

Por último, hago remisión a un estudio realizado por mí en el 2017 sobre un caso de reubicación post-impacto donde se manifestaron dos comportamientos resilientes en los dos grupos divididos de la comunidad Barranca Grande luego de ser reubicada en la localidad Barranca Nueva, en Veracruz, México. Las personas que permanecieron en Barranca Grande mostraron un comportamiento conservativo al rehabilitar la agricultura, reconstruir el asentamiento dañado y restablecer la dinámica social.

En cambio, los reubicados desplegaron un comportamiento adaptativo mediante las intervenciones físico-espaciales realizadas al nuevo hábitat; dígase: la construcción de lugares colectivos culturalmente relevantes (templo, salón social y viveros de café) que les exigió del fortalecimiento de las redes sociales, la cooperación y responsabilidad, así como la modificación de sus viviendas para recrear ciertas actividades cotidianas y tradicionales del ámbito doméstico -cocina de leña, cultivos en el patio, portal para las celebraciones- (Montero, 2018).

En definitiva, estos estudios evidencian diversos comportamientos resilientes de grupos sociales con capacidades y recursos variados, de modo que reconocer y explicar la resiliencia social no es tarea fácil. Lo variopinto de la comprensión y aplicación del concepto lo hace difícil. Sin embargo, es posible acceder a una claridad conceptual con la aplicación de la heurística del reconocimiento que permita saber la naturaleza y función de la resiliencia social.

Esta heurística busca explicar, en general, el comportamiento de los agentes sociales, por lo que resulta ajustable para enfocar metodológicamente la identidad de la resiliencia social empíricamente constatable. Ante la pregunta: ¿Es la comunidad $X$ más resiliente que la $Y$ ?; debemos saber si cada una primeramente lo es sirviéndonos de los pocos datos que tengamos. Si las capacidades o recursos son esos datos, no podemos contestar; en cambio, si observamos los dos comportamientos, entonces sí podremos responder con base en la crisis superada.

La heurística del reconocimiento permite hacer inferencias cuando no es posible razonar lógicamente y estadísticamente sobre criterios fácilmente verificables; más bien, son inferencias por valores y motivos personales regidos por un ejercicio de reconocimiento que reduce los criterios u opciones. No obstante, este método no desestima la racionalidad de ciertos rasgos o indicios indicativos de fiabilidad; por lo que, para hacer inferencias precisas, es imprescindible tener algún conocimiento fehaciente de ciertos criterios y rasgos luego de correlacionar lo reconocido y aquello que se quiere saber (Gigerenzer, 2008).

Aplicar este método para el reconocer a la resiliencia social exige de la identificación y la contextualización, pues, como bien indicó Gigerenzer (2008, p. 123): "El reconocimiento es la capacidad para distinguir lo novedoso de lo experimentado antes [...]. La capacidad de reconocimiento está adaptada a la estructura del entorno". Así, considero que el sustrato lógico del método es espontáneo y no aritmético, por ello me apoyo en las pautas del reconocimiento filosófico desentrañadas por Ricoeur (2006) para darle cierta solidez a la espontaneidad.

Según reflexionó Ricoeur (2006), con base en Descartes y Kant, reconocer tiene una carga en el identificar, y este a su vez, en el distinguir y el relacionar. Para identificar a la resiliencia social hay que distinguir entre sus definiciones y los conceptos asociados: resistencia, adaptación y transformación. De plano, podemos distinguir dos momentos diferentes que se relacionan: uno cuando el sistema trabaja para sus capacidades, recursos o propiedades, y otro cuando actúa para cambiar el estado negativo en que se encuentra. Por tanto, imposibilitado de discriminar entre definiciones y conceptos asociados, pues al parecer todos constituyen a la resiliencia social, identifico el paso del primer momento al segundo como un proceso necesario.

Ahora es preciso contextualizar este proceso identificado, pues la relatividad de la realidad lo exige. Contextualizar es poner en un ámbito de realidad y sentido ese algo identificado para que sea de una manera particular; por tanto, considero que son las experiencias individuales y sociales de los seres humanos eso que contextualiza al proceso capacidades-acciones -sintetizado así para abreviar. Licencio entonces la propuesta de Koselleck (1993; 2001) de: campo de experiencias más horizonte de expectativas para el propósito metodológico que me ocupa. 
El contexto de la resiliencia social es el marco de expectativas nuevas e individuales que pueden ser también compartidas por una generación histórica, sumadas a las experiencias estructurales (transgeneracionales y culturales), y cuales expectativas constituirán experiencias adicionales en su tiempo. Así, todo lo aprendido, construido, organizado y reservado para períodos de desastres constituye experiencias heredadas y vividas, como también expectativas de seguridad y fortaleza ante futuros desastres.

La propuesta que a partir de aquí pretendo validar es que la resiliencia social puede ser reconocida dentro del movimiento procesual que determina el paso de capacidades, recursos o propiedades a acciones preservativas, adaptativas y/o transformativas del sistema social, en contraposición a la producción, el incentivo y fortalecimiento de capacidades, recursos y propiedades que no puedo conocer fuera del comportamiento práctico. A consecuencia, ella puede ser asumida como un fenómeno social en un contexto particular según las experiencias y expectativas de las comunidades o sociedades; un contexto determinado por el encuentro entre el sistema social y el sistema natural, o sea, un contexto dado por el conflicto entre agentes.

Para tal pretensión retomo los postulados analíticos de Inmanuel Kant y Georg Simmel porque Kant (2009) fue el primero en evidenciar el papel del ser humano en la determinación de la realidad fenoménica de las cosas al momento de destacar las necesarias acciones perceptivas e intelectuales en la definición de las cosas aparentes desde un ejercicio de consciencia ineludible.

Por su parte Simmel (1927) abordó la determinación del fenómeno en la dimensión social de la realidad desde la génesis del conflicto; conflicto no únicamente negativo, sino también positivo para ambas partes en interacción. Para él la lucha constituía ese conflicto social con implicaciones generadoras y re-generadoras de la realidad social. De modo que todo conflicto con acción humana involucrada es per se un fenómeno social con registros negativos y positivos.

\section{KANT Y SIMMEL PARA LA RESILIENCIA SOCIAL}

Kant (2009) ya había evidenciado el paso de capacidades a acciones en el proceso epistémico de la realidad. Primero refirió que de las intuiciones puras (temporales y espaciales) se partía para conocer los objetos, lo cual permite asumirlas como capacidades epistémicas del ser humano. Luego asintió que hay ejercicios perceptivos e intelectuales posibilitados por esas capacidades específicas.

En este sentido epistémico, es que la resiliencia social se nos desvela como un fenómeno identificable tras observar el comportamiento de afrontamiento y superación de las personas y sus efectos positivos. No es un objeto pensado en abstracto para darle sentido a un problema del pensamiento. Por eso, a partir del fenómeno es que conceptualizamos tal comportamiento intuido desde una identificación sensitiva e intelectual.

Tal objetividad identificada en la resiliencia social reside en la entitividad que le confiere la sistematización social, o sea, el paso de capacidades, recursos o propiedades a acciones en el contexto de sistematicidad del sistema social que se objetiva en sus acciones e interacciones estructurales y organizacionales. Dentro de esta identificación kantiana del objeto, la resiliencia social es un fenómeno intuido a partir de conocer las acciones sociales que provocan el cambio, no es intuido en aquellos elementos previos no perceptibles $u$ ocultos que, preponderados en la definición, considero que provocan su disociación nouménica.

Ahora bien, posicionado en esta noción del noúmeno que para Kant (2009) remitió a la cosaen-sí diferente a la cosa-para-la-conciencia percibida y entendida, digo que la resiliencia social percibida en la realidad fenoménica no puede ser entendida como algo diferente a un fenómeno social. Es decir, aquello distinto a esta cosa que percibo o intuyo en su apariencia (comportamiento resiliente) es algo que esta cosa no es (capacidad, recurso o propiedad oculta); le pertenece a su amplitud de ser (resiliencia trascendente) que no se me muestra en su absolutidad; ella estásiendo en un plano irreconocible para mí al momento de percibirla o intuirla. Por tanto, aquello que no identifico y que representa lo que la resiliencia identificada no es, es algo nouménico.

La susceptibilidad de la resiliencia social a nuestra observación, comprensión e interpretación -evidente en los casos antes citados- nos confirma su naturaleza fenoménica y su función causal. Si la continuamos asumiendo únicamente como 'capacidad de-', 'recurso para-' o 'propiedad por la cual-', entonces estaríamos ignorando su característica procesual y con ello limitaríamos entre sus imposibilidades al fenómeno social reconocido. Esto quiere decir que, constreñida en estos elementos ocultos e imperceptibles, la resiliencia social como fenómeno no sería posible porque no 
podría pasar de potencias (capacidades) a actos (acciones) y, por consiguiente, nunca hubiéramos podido reconocerla en los sistemas sociales cualificados como resilientes por sus comportamientos específicos, lo cual dejaría exclusivamente a dicha noción de resiliencia en un plano nouménico, sin actos que la desoculten.

En la teoría de la acción, solo se llega al acto si hubo una potencia o capacidad de ello. Por tanto, no es un error remitir regresivamente a las capacidades, recursos o propiedades del sistema social luego de constatar las acciones resilientes inherentes a un fenómeno social reconocido. Sin embargo, cuando desde aquella enunciación nouménica definimos a la resiliencia social, limitamos su ocurrencia en la realidad operativa y caemos en la disociación nouménica que desestima o ignora lo práctico del fenómeno. A consecuencia, su operación no es del todo efectiva en el comportamiento resiliente de cambio.

Resumiendo: tanto los ejemplos de resiliencia en ecosistemas son fenómenos naturales perceptibles, registrables y comprendidos (Holling, 1973; Walker et al., 1997; Gunderson, 2000), como los casos de resiliencia en sistemas sociales son fenómenos sociales (Carpenter, 2014; Herrera \& Rodríguez, 2016; Montero, 2018). Son fenómenos sociales porque ocurren cuando los actores sociales deciden actuar colaborativamente para llevar a positivo el estado adverso o crítico en que viven luego del desastre.

Esta decisión para actuar es propia de la especie humana que además de ser social es política, lo cual le abona más incertidumbre a la seguridad de que una comunidad capacitada para afrontar y superar una situación crítica cualquiera, y aprender de ello, pueda llevar a cumplimiento efectivo dichas acciones. Las conductas, inclinaciones e incentivos de los actores sociales son diversos, por lo que no se puede dar fe de algo sin que haya un comportamiento que lo valide. En este sentido, la resiliencia social asumida como un fenómeno social permite interpretar las conductas de los actores sociales relacionados entre sí por las acciones que desempeñan en un contexto social caracterizado por las condiciones del medio, la historia y el horizonte de futuro, o sea, por las experiencias y expectativas

A propósito, considero que la resiliencia social posee un esquema fenomenológico similar al del conflicto, pues, a primera instancia, comprendemos su naturaleza a partir de las acciones y su función, desde sus resultados; y en segunda instancia, ella sucede cuando un agente perturbador (ej. una amenaza natural) ha impactado en el sistema social vulnerable y activo que intenta reaccionar de alguna manera. Por demás, en el conflicto suceden especiales experiencias y expectativas que compaginan perfectamente con las capacidades, recursos o propiedades a priori para reaccionar y con las finalidades perseguidas.

Me apego al esquema teórico de la lucha cual fenómeno socializante desarrollado por Simmel (1927) quien la reconoció como una ramificación particular de algo más general -el conflicto a mi parecer-, algo que excede la distinción antagónica de elementos o ideas dentro de una noción binaria de contrarios que estructuran nuestro entendimiento de la realidad. Para él, tales elementos binarios constituyen una unidad copulativa de esas nociones opuestas por donde oscila la vida, aun cuando no lo entendamos de esa manera.

La lucha es un fenómeno sociológico y, a diferencia de la creencia común, no es solo negativa, sino también positiva porque en ella las formas sociales adquieren un orden particular, se enardecen los sentimientos individuales y colectivos, se nutre de vivacidad las relaciones sociales, y se alcanza una conciencia de las fuerzas propias y un reconocimiento de las capacidades (Simmel, 1927). Por ende, el conflicto posibilita que al menos una de las partes reconozca sus capacidades, recursos y propiedades, que gane confianza para futuros hechos y refuerce sus redes sociales, tal cual sucede en la resiliencia social.

Para Simmel (1927) la lucha es una relación que le solicita al entendimiento humano un ejercicio de abstracción lógica para extraer los factores antagónicos que le figuran y que solamente podemos escindir ya constituida una relación. Así, los grados de superioridad y sugestión que ella produce en cada uno de sus contrincantes únicamente pueden ser advertidos al identificar la fuerza de uno en contra de la debilidad del otro y llegar entonces a tal escisión.

La fortaleza y la debilidad, cuales atributos inferibles, no son visibles sin esa relación. El conflicto evidencia acciones que colisionan y que en la lucha son recíprocas, pero no evidencia ciertas cualidades internas de los agentes conflictuantes. En la resiliencia social tampoco las 
capacidades pueden ser identificadas si no hay acciones expresas que remitan a ellas. Así pues, tanto la fortaleza, como las capacidades, recursos o propiedades que les atribuimos a la resiliencia, emergen a posteriori de que esta ya forme parte del conflicto.

Por otro lado, son las acciones las que exteriorizan esos aspectos profundos de las pasiones humanas que son motor de lo activo. Esto Simmel (1927) lo reconoció en la lucha, al plantear que los sentimientos se avivan e impulsan la acción desde el ánimo. En la lucha hay una acomodación interna de los sentimientos que provee las fuerzas necesarias para actuar sobre la situación de conflicto que debe ser explotada o combatida, soportada o abreviada; es una lucha que surge de pasiones internas que encuentran adecuo en el oponente.

También la resiliencia social concuerda con ese ánimo impulsor de las acciones colectivas en la lucha, pero, a diferencia, sí encuentra excitación en las amenazas futuras que les exigen a la sociedad de la recuperación, la mejora y el bienestar. La posible ocurrencia de la amenaza excita los sentimientos para cambiar el estado crítico actual y reducir su nocividad, lo cual obliga a lograr ese otro estado favorable para afrontar nuevos conflictos.

Esta preparación posterior a la agresión primera y anterior a las agresiones futuras es evidencia del carácter procesual de la resiliencia social. Es un proceso de cambios inevitables para las poblaciones que han vivido un desastre, donde los ánimos compartidos colectivamente se inflaman ante la posibilidad de cambiar ese estado crítico a otro estado fructuoso y benéfico. Así, en esta caldera anímica, los sentimientos son intensificados y sustituidos por otros, tal y como la inseguridad pasa a ser confianza, el temor, a ser valentía, y el sentimiento de indefensión trasmuta en protección social.

"La lucha, en efecto, plantea condiciones previas y produce modificaciones y adaptaciones necesarias para el mejor desarrollo del conflicto" (Simmel, 1927, p. 69). Similarmente, en la resiliencia social ocurren cambios perfectamente visibles en la organización y función del grupo social, y otros invisibles, en las capacidades y propiedades del sistema. El sistema social en resiliencia convierte sus debilidades en fortalezas, da nuevo orden a las redes sociales con presencia o no del liderazgo y gestiona funciones extraordinarias para las instituciones y actores sociales participantes.

Orden, función y fortaleza pasan a ser aspectos fundamentales en la participación de actores sociales e instituciones en la resiliencia social cual fenómeno coyuntural dentro del conflicto que representa el proceso de desastre en general. De suyo, entonces, tal como en la lucha que hay apoyo y solidaridad interna, la resiliencia también precisa de ello. Según Simmel (1927) en los grupos sociales bien organizados y unidos, las disensiones intestinales son mejormente soportadas porque las fuerzas sintéticas -integradoras- contrarrestan las antitéticas -disolventes. En estos grupos las uniones producen solidaridad entre los elementos sociales, de modo que los daños de la lucha pueden ser subsanados con relativa facilidad. La lucha y sus daños pueden ser fácilmente atendidos por el Estado o dejados a la suerte de sus bandos en disputa sin representar amenazas para el armazón político.

Esto es un procedimiento recurrente para pequeños conflictos que responde al principio social del aislamiento de las partes en lucha. Ellas deben resolver sus desavenencias y soportar, por demás, los daños generados sin afectar al todo (Simmel, 1927). Coincidentemente, en la resiliencia social también ocurre esto, pues su tratamiento político ha servido para refundar subjetividades patológicas en el peligro y en la inseguridad ante el mundo natural y antrópico, lo cual deposita en los individuos el peso de la responsabilidad de protegerse a sí mismos (Evans \& Reid, 2016), al tiempo que es un concepto disociativo de los problemas medulares de la vulnerabilidad para la agencia estatal: la pobreza y la desigualdad social (Macías, 2015).

El orden, la función y la fortaleza dependen de la colaboración del Estado e instituciones competentes en la resiliencia social cual fenómeno social definido por el proceso de acciones y comportamientos resilientes. Contrario a la opinión de Cannon y Müller-Mahn (2010) de una equívoca noción de fenómeno social para la resiliencia desprovista de la relación de poder que el esquema vulnerabilidad-resiliencia entraña, considero que la resiliencia social solo puede ser planificada y efectiva en su objetivo si es asumida como el fenómeno social que es. Su pragmatismo es anterior y posterior al evento catastrófico donde tienen partido esas vulnerabilidades gestadas en la relación de poder. La condición vulnerable previa que incluye la etapa de preparación resiliente en su favor o detrimento es un fenómeno social particular que se conecta con la resiliencia social 
activa posterior cual fenómeno social de acciones y cooperaciones necesarias. Entre uno y otro hay traspasos de elementos que evidencian al desastre como un proceso multiespacial y diacrónico.

\section{DISCUSIÓN CONCLUSIVA}

De ahí que, en la resiliencia social haya un paso impulsivo de las capacidades, características, atributos, recursos o propiedades del sistema social -a saber: 1) recursos cognitivos y de aprendizaje para la planeación y reorganización, 2) potencialidades de redes sociales eficaces para el afrontamiento, 3) conexión del grupo social con el lugar, 4) posibilidades económicas y gubernamentales para lograr el cambio y 5) recursos culturales que a manera de hábitos recuperables propulsan a la superación de la crisis (Marshall \& Marshall, 2007; Ross et al., 2010; Faulkner et al., 2018; Montero, 2018)-, hacia un determinado comportamiento social definido por sus acciones resilientes de afrontamiento, conservación resistente, adaptación y/o transformación (MCEER, 2007; Keck \& Sakdapolrak, 2013; Uriarte, 2013; Carpenter, 2014; Herrera \& Rodríguez, 2016; Montero, 2018).

Esto demuestra que las investigaciones han estado encaminadas a estudiar tanto las capacidades, recursos y propiedades de los sistemas sociales dentro de sí mismos o dentro de sistemas complejos como los socio-ecológicos (Holling, 1973; Walker et al., 1997; Gunderson, 2000), al igual que han descrito los procedimientos y actividades colectivas en una fase de recuperación después del desastre. De modo que los primeros estudios explican el fenómeno social de gestión de riesgo, mientras que los segundos abordan el fenómeno social de rehabilitación, reconstrucción y reubicación principalmente. En unos hubo pretensiones de cuantificar, estandarizar, monitorear, evaluar y medir a la resiliencia social a manera de explicarla, mientras que los otros evidenciaron la realidad fáctica de lo que ella es y puede hacer. Estos cimentaron su epistemología en la observación objetiva de las acciones y sus efectos, mientras que aquellos estuvieron circunscriptos en la noción de capacidad con el uso de métodos de exploración subjetiva como la encuesta y las entrevistas.

Cuando en el proceso de desastre sucede el paso de un fenómeno a otro, podemos reconocer a la resiliencia social como un proceso configurado por experiencias de desastres y superaciones, y expectativas de mejora y bienestar con implicaciones positivas en lo social, cultural, político, económico y tecnológico de las sociedades. Este es un proceso donde además ocurren traspasos de elementos que hacen más fuertes o débiles a las sociedades, más vulnerables o combativas en un contexto de conflicto reiterado.

El conflicto se da cuando agentes naturales y agentes sociales interaccionan repetidamente de maneras y grados diversos, tal y como demostraron Holling (1973), Walker et al. (1997) y Gunderson (2000) sobre la perturbación humana en los ecosistemas resilientes, y como (Carpenter, 2014; Herrera \& Rodríguez, 2016; Montero, 2018) evidenciaron sobre las reacciones humanas ante las perturbaciones naturales.

El proceso de desastre, cual conflicto, produce efectos negativos y otros positivos, la resiliencia social es un aspecto positivo de ello dado que proporciona aprendizaje y ajustes organizacionales y funcionales que fortalecen al sistema social. No obstante, solamente podremos saber lo fuerte o débil que ella es en su momento efectivo como fenómeno social reactivo. Según el impacto benéfico de las acciones resilientes es que podemos determinar su fuerza o debilidad y conocer las capacidades, recursos y propiedades que la sociedad posee y necesita reforzar para futuros desastres.

Por ello el comportamiento resiliente, así como su reforzamiento y desarrollo constante necesitan del impulso de las ánimos y sentimientos sociales, los cuales son motores de ese comportamiento ante las crisis, pero también, respuestas al estímulo de futuros desastres que se materializa en la gestión del riesgo, la preparación de acciones y la planificación organizacional y funcional del sistema social particular.

Por consiguiente, no propongo abandonar la noción de capacidad regente hasta hoy, sino incluir la noción activa de comportamiento y fenómeno, puesto que lo primero ha servido para gestionar y planificar los activos o recursos sociopolíticos y económicos hacia la capacitación de los grupos sociales, mientras que lo segundo hace obliga a hacer posible tal aspiración desde la colaboración popular-institucional que, en caso contrario, provocaría otras crisis dentro del proceso de desastre.

Lo logrado en este artículo teórico es el reconocimiento de la resiliencia social como fenómeno social dentro del proceso de desastre como conflicto complejo que posee fases socio-naturales y 
socio-institucionales que pueden deparar en crisis sociales. De aquí, la explicación de ella, o sea, los estudios sobre su operatividad, gestión y planificación adquieren nuevo rumbo que otros analistas recorrerán.

Por mi parte propongo adoptar el verbo resilir, proveniente del anglo: to resile, para aportar esa denotación activa necesaria. Será este, siguiendo el tono kantiano, un concepto derivado de la síntesis realizada a partir del reconocimiento de múltiples acciones resilientes; no remite a una acción específica, sino a un conjunto de ellas.

De esta manera, la teorización de la resiliencia social podría arrancar desde la realidad fenoménica, evitando ciertas desviaciones de sentido y errores de aplicación que obstruyen su operacionalidad. Esto afianzaría la responsabilidad de los actores sociales e instituciones gubernamentales a actuar solidaria y cooperativamente para cambiar las situaciones críticas a estados positivos.

El resilir quedaría instaurado como la mayor responsabilidad que los actores sociales e instituciones gubernamentales deben tener en procesos de desastres; pues, dentro de este proceso, cual conflicto que es, resilir y dimitir comparten lugar en las voluntades que tanto impelen los ánimos combativos como la indolencia o la apatía. Por tanto, esta es una responsabilidad absoluta que imperiosamente requiere de la colaboración y del respeto a las diversas maneras de actuar, que necesita de fomentar y fortalecer las capacidades, recursos y propiedades sociales, al tiempo que apoya y coadyuva las acciones posteriores. Entonces, los integrantes sociales deben hacer prevalecer al resilir y los Estados deben estar comprometidos a ello.

En suma, la resiliencia social, cual fenómeno social, requiere del previo conocimiento del conflicto y de sus agentes interactuantes; se debe conocer cómo las experiencias y expectativas configuran el contexto del proceso de desastre para el resilir que supera la actual crisis y prepara para las siguientes. En este sentido, la resiliencia social es tanto un proceso de capacidades impulsoras de acciones, como el resultado de la producción y/o fortalecimiento de capacidades, recursos y propiedades por esas acciones resilientes. Ocurre así una modificación o ajuste del sistema social que evidencia recuperación, adaptación y/o transformación de sus elementos organizacionales y funciones.

El resilir gana relevancia entonces. Su significado es: 'el participar activamente en el fenómeno social de resiliencia y ser responsable de su proceso y resultado'. Por consiguiente, en procesos de desastre, el resilir es gestionable en la fase preventiva y coadyuvable en la etapa de recuperación post-impacto, presente en la reconstrucción y en la reubicación poblacional. En él, la responsabilidad y el comprometimiento del Estado con los agentes sociales permiten cambiar lo negativo de las situaciones críticas a estados positivos que posibiliten afrontar futuras catástrofes y prevenir desastres.

\section{REFERENCIAS}

Abend, G. (2008). The Meaning of 'Theory'. Sociological Theory, 26(2), 173-199. https://doi. org/10.1111/j.1467-9558.2008.00324.x

Ainuddin, S. \& Routry, J.K. (2013). Earthquake hazards and community resilience in Baluchistan. Natural Hazards, 63, 909-937. https://doi.org/10.1007/s11069-012-0201-x

Arévalo, M.L. (2012). Prácticas espaciales y socioeconómicas en la Ciudad Rural Sustentable Nuevo Juan de Grijalva (Tesis de maestría). Chiapas, México: CIESAS.

Briones, F. (2010). Inundados, reubicados y olvidados: Traslado del riesgo de desastres en Motozintla, Chiapas. Revista de ingeniería, 31, 132-144. http://ref.scielo.org/dw74vp

Carpenter, A. (2014). Resilience in the social and physical realms: Lessons from the Gulf Coast. International Journal of Disaster Risk Reduction, 4(3), 290-301. https://doi.org/10.1016/j. ijdrr.2014.09.003

Contreras, Y. del C. \& Beltrán, M. (2015). Reconstruir con capacidad de resiliencia: el caso histórico de la ciudad de Constitución y el sitio del desastre del terremoto y tsumani del 27 de febrero 2010. Revista INVI, 30(83), 79-115. http://dx.doi.org/10.4067/So718-83582015000100003

Cutter, S.L., Barnes, L., Berry, M., Burton, Ch., Evans, E., Tate, E. \& Webb, J. (2008). A place-based model for understanding community resilience to natural disasters. Global Enviromental Change, 18 , 598-606. https://doi.org/10.1016/j.gloenvcha.2008.07.013 
Department for International Development of the United Kingdom DFID. (2011). Defining Disaster Resilience: A DFID Approach Paper. Londres: DFID. Recuperado de https://www.gov.uk/ government/publications/defining-disaster-resilience-a-dfid-approach-paper

Evans, B. \& Reid, J. (2016). Una vida en resiliencia. El arte de vivir en peligro (Trad. V. Altamirano). Ciudad de México: Fondo de Cultura Económica.

Faulkner, L., Brown, K. \& Quinn T. (2018). Analyzing community resilience as an emergent property of dynamic social-ecological systems. Ecology and Society, 23(1), 24. https://doi.org/10.5751/ ES-09784-230124

Forés, A. \& Grané, J. (2008). La resiliencia. Crecer desde la adversidad. Barcelona: Editorial Plataforma.

Gigerenzer, G. (2008). Decisiones instintivas: la inteligencia del inconsciente (Trad. J. Soler). Barcelona: Ariel.

Gunderson, L.H. (200o). Ecological Resilience-in Theory and Application. Annual Review of Ecology and Systematics, 31, 425-39. https://doi.org/10.1146/annurev.ecolsys.31.1.425

Gunderson, L.H. \& Holling, C.S. (2002). Panarchy. Understanding transformations in human and natural systems. Washington DC: Island Press.

Herrera, G. \& Rodríguez, G. (2016). Resiliencia y turismo: El caso de la ciudad de Baños de Agua Santa Ecuador. Holos, 32(3), 229-250. http://dx.doi.org/10.15628/holos.2016.4303

Holling, C.S. (1973). Resilience and Stability of Ecological Systems. Annual Review of Ecology and Systematics, 4, 1-23. Recuperado de https://www.jstor.org/stable/2096802

Kant, I. (2009). Crítica de la razón pura (Trad. M. Caimi). Ciudad de México: Fondo de Cultura Económica.

Keck, M. \& Sakdapolrak, P. (2013). What is social resilience? Lessons learned and ways forward. Erdrunde, 67(1), 5-19. http://doi.org/10.3112/erdkunde.2013.01.02

Koselleck, R. (1993). Futuro pasado. Para una semántica de los tiempos históricos (Trad. N. Smilg). Barcelona: Paidós.

Koselleck, R. (2001). Los estratos del tiempo: estudios sobre la historia (Trad. D. Innerarity). Barcelona: Paidós-Universidad Autónoma de Barcelona.

Ley General de Asentamientos Humanos, Ordenamiento Territorial y Desarrollo Urbano. Diario Oficial de la Federación, México, 28 de noviembre de 2016.

Ley General de Protección Civil. Diario Oficial de la Federación, México, 6 de junio de 2012, Reformada el 7 de abril de 2017.

Macías, J.M. (2009). El modo de orientación de las reubicaciones. En. J. M. Macías (coord.), Investigaciones evaluativas de reubicaciones humanas por desastres en México (pp. 49-98). México D.F.: CIESAS.

Macías, J.M. (2015). Crítica de la noción de resiliencia en el campo de estudios de desastres. Revista Geografía Venezolana, 56(2), 309-325.

Marshall, N.A. \& Marshall, P.A. (2007). Conceptualizing and Operationalizing Social Resilience within Commercial Fisheries in Northern Australia. Ecology and Society, 12(1), 1. http://doi.org/10.5751/ ES-01940-120101

McAdam, J. (2015). Las lecciones de anteriores reubicaciones y reasentamientos planificados. Revista Migraciones Forzadas, 49, 33-32. http://hdl.handle.net/10045/47537

MCEER. (2007). Engineering resilience solutions from earthquake engineering to extreme events. Buffalo, NY: Multi-disciplinary Center for Earthquake Engineering Research. Recuperado de http:// www.eng.buffalo.edu/mceer-reports/o8/o8-SPo9.pdf

Montero, J.L. (2018). El acomodo espacial como acción de los reubicados hacia el logro de la vivienda adecuada en Barranca Nueva, México, 2010-2018 (Tesis de maestría). Xalapa, México: Universidad Veracruzana. Recuperado de https://cdigital.uv.mx/handle/1944/49451

Oliver-Smith, A. (1991). Successes and Failures in Post-Disaster Resettlement. Disasters, 15(1), 12-23. http://doi.org/10.1111/j.1467-7717.1991.tboo423.x

Oliver-Smith, A. (1994). Reconstrucción después del desastre: una visión general de secuelas y problemas. En A. Lavell (comp.), Al norte del Río Grande, Ciencias Sociales, Desastres: una perspectiva norteamericana (pp. 25-44). Bogotá: Tercer Mundo Editores. 
Prieto, E.A. (2013). Resiliencia y panarquía: claves para enfrentar la adversidad en sistemas sociales. Multiciencias, 13(1), 23-29.

Ricoeur, P. (2006). Caminos del reconocimiento: Tres estudios (Trad. A. Neira). México D.F.: Fondo de Cultura Económica.

Ross, H., Cuthill, M., Maclean, K., Jansen, D. \& Witt, B. (2010). Understanding, Enhancing and Managing for Social Resilience: Opportunities in Far North Queensland. Cairns, Australia: Reef and Rainforest Research Centre.

Simmel, G. (1927). La lucha. En G. Simmel. Sociología. Estudios sobre las formas de socialización (Trad. J. Pérez) (pp. 9-99). Madrid: Revista de Occidente.

UNDRR (2020). Resilience. UNDRR [web]. Recuperado de https://www.undrr.org/terminology/ resilience

Uriarte, J. de D. (2013). La perspectiva comunitaria de la resiliencia. Psicología política, 47, 7-18.

Walker, B.H., Langridge, J.L. \& Mcfarlane, F. (1997). Resilience of an Australian savanna grassland to selective and nonselective perturbations. Australian Journal of Ecology, 22, 125-135. https://doi. org/10.1111/j.1442-9993.1997.tboo651.X

Warner, K., Kälin, W., Martin, S. \& Nassef, Y. (2015). Los planes de adaptación nacionales y la movilidad humana. Revista Migraciones Forzadas, 49, 8-9. http://hdl.handle.net/10045/47527 